\title{
DETERMINING HIGHWAY CORRIDORS
}

November 28, 2011

\author{
Eusebio Angulo ${ }^{1}$, Enrique Castillo ${ }^{2}$ \\ Ricardo García-Ródenas ${ }^{1}$ and Jesús Sánchez-Vizcaíno ${ }^{3}$ \\ ${ }^{1}$ Department of Mathematics, \\ University of Castilla-La Mancha, Spain \\ ${ }^{2}$ Department of Applied Mathematics and Computational Sciences, \\ University of Cantabria, Spain \\ ${ }^{3}$ Department of Civil Engineering, \\ University of Castilla-La Mancha, Spain
}

\begin{abstract}
In the highway development process, the first planning stage is that of selecting a corridor along which the highway is to pass. Highway corridor selection represents a multicriteria decision process in which a variety of social, enviromental and economic factors must be evaluated and weighted for a large number of corridor alternatives. This paper proposes a demand-based approach to provide a set of potential corridors. The problem is formulated as a continuous location model which seeks a set of optimal corridors with respect to the demand of potential users while satisfying budget constraints. This model uses geographical information in order to estimate the length-dependent costs (such as pavement and construction cost) and the cost of earth movement. A procedure for finding the best local minima
\end{abstract}


of the optimization model is proposed. This method is tested using the Particle Swarm Optimization algorithm, two algorithms of the Simulated Annealing type and the Simplex Nedelmar method. An application using the Castilla-La Mancha's geographic database is presented.

Keywords: Highway corridor location - Demand covering - Heuristics.

\section{Introduction}

Highway corridor location takes up a huge amount of resources but provides important savings in cost and high improvements in performance of these elements, which are expected to provide the best possible service for the geographical distribution of demand. The location of highway corridors is a fundamental part of any highway design project and has produced important tools and studies over the years.

In this paper the problem of determining highway corridors in a geographical region including rural or mixed rural-urban areas is addressed. It is assumed that the level of congestion is negligible. The uncongested highway design and planning consists of three successive stages as shown in Figure 1. In the highway corridor selection stage several potential corridors are generated and analyzed in terms of user's benefits, regional development benefits, environmental and social benefits, costs, etc. The generation of these alternatives requires analytic tools which allow these corridors to be located in space in accordance with difficult to deal with factors like potential demand in the geographical 
area studied, and the available budget, from which an evaluation of the economic effects on the region, the environmental effects and the community concerns can be made. Following this stage is the Highway Alignment Optimization stage (HAO), whose input data are the highway endpoints and the estimated demand between the endpoints. These inputs are used at this stage to calculate the alignment which minimizes a certain combination of costs, such as the operator costs plus the user costs for the estimated demand, satisfying design and operational constraints in a bounded search space. As a result, a polygonal is obtained which defines the initial route on which bridges, junctions and other important elements are located. Finally, in the geometric design stage this polygonal is adjusted to satisfy all current highway construction laws and associated constraints.

We have reviewed a large collection of papers in the existing literature addressing the highway design and planning problems regardless of congestion. Table 1 shows some examples of these studies. The papers reviewed depending on the problem being addressed are grouped according to the different categories shown in Figure 1.

This paper belongs to the first group, i.e. it deals with the highway corridor selecting problem for uncongested networks. Existing combinational optimization models, such as ([Solanki et al., 1998]; [Petersen, 2002]; [Drezner and Wesolowsky, 2003]; [Kim et al., 2008]; [Zhu et al., 2010]) calculate an optimal sequence and timing of improvements for a highway corridor, subject to the budget constraints. These models optimize the highway network layout and the roadway configurations of an initial existing corridor. In this paper we describe a model for locating new highway corridors so as to maximize demand 
capture for a construction cost lower than the available budget. This problem is formulated as a continuous location model and allows a great flexibility in the definition of the costs involved in the alignment. The Geographic Information Systems (GIS) permits to measure specific costs in the study area which represent the construction costs as a function of the geological characteristics of the terrain, the cost of land purchase, earth movement and/or the need to include junctions or bridges. From these estimates the model works with a bivariate linear interpolation function and defines the costs as the curvilinear integral with respect to the interpolated cost function. The results obtained with this model are essentially the data required by the HAO, such as the endpoints of the highway and an estimate of the traffic demand to be served, which is necessary to define the user costs in a later planning stage. Due to the complexity of the HAO, highway planners often deal with this problem using a two-stage approach to select a highway route. In the first stage, they solve the horizontal alignment problem and in the second stage they design the vertical alignment based on the horizontal resulting alignment (see [Parker, 1977]; [Cheng and Lee, 2006]). In the existing literature, other models have appeared in which the three-dimensional (3D) alignment (horizontal and vertical) problem is solved directly (see, for example, [Chew et al., 1989]; [Jong and Schonfeld, 2003]; [Cheng and Lee, 2006]; [Kang et al., 2009]). As it can be seen in Table 1, most of the works dealing with the HAO problem are solved by means of genetic algorithms and Geographic Information Systems (GIS). These studies have been sequencially improved by their authors, by considering more and more factors. This has permited better solu- 
tions as a consequence of the substantial improvement of computational tools and power. For example, [Jha and Schonfeld, 2003] add to previous work ([Jha and Schonfeld, 2000b]; [Jha and Schonfeld, 2000a]; [Jha et al., 2001]; [Jha, 2001]; [Jha, 2003];) the maintenance costs. [Kim et al., 2004a] and [Kim et al., 2004b] improve previous studies by taking into account the intersection costs. [Jha and Kim, 2004] consider the traffic demand in the optimization problem. [Kim et al., 2005] add to the construction cost other factors, such as the topography, socio-economical data of the area being studied, geological and ecological data and the type of terrain. [Kang et al., 2009] centre mainly on the computation time and the quality of the optimal solution. These models search for the optimal solution by minimizing a total cost function having alignment-sensitive cost components and satisfying design, environmental, and other geographic constraints. A comprehensive revision of the different costs involved in the objective function of the $\mathrm{HAO}$ problems is given by [Kang, 2008] and the many references contained therein. Although these models have acquired a high level of sophistication they all assume that the endpoints of the highway and the demand are already known. To carry out a review of this subject is beyond the scope and aims of this paper and interested readers may consult the paper [Kim et al., 2005].

The rest of the paper is organized as follows. Section 2 presents a continuous location model and explains the building cost function and the corridor selection criterion. Section 3 proposes four heuristic algorihtms for solving the location model. In Section 4, computational experiments are carried out on a real case study. The last section of the 
paper provides some conclusions.

\section{A continuos location model for selecting a highway}

\section{corridor}

Traditionally, the highway agencies use construction costs, users' costs, economic effects on the region, environmental effects and community concerns as criteria for the selection of highways.

Government agencies invest in new highways as a means of stimulating economic growth and increasing competitiveness in a particular region. These goals should be guided by analytical tools which allow us the impact of the new transport infrastructures to be measured. A key aspect of social utility to be measured is the demand they will bear. A perspective adopted in many public highway agencies is to select the highway which can be undertaken with the available budget in such a way as to maximize the use of the new infrastructure. This is the main perspective taken in this paper. In this section we describe an optimization model with this objective. Firstly we introduce a procedure for estimating the construction costs of a highway, then we design a procedure for estimating the highway demand as a function of its location, and finally we incorporate both methods into an optimization model. 


\section{Building the cost function}

A corridor is represented by a rectangle (see Figure 2) which is completely defined by points $A, B$ and the width $\delta$. Points $A$ and $B$ are key elements since they determine the endpoints of the highway for the HAO problem while the width of the rectangle, $\delta$, may be chosen ad hoc, taking a width sufficient to enclose the optimal alignment. We consider that the segment linking points $A$ and $B$ is an approximation to the alignment it is intended to build.

In order to choose the best location for the new corridors, in addition to other factors, we need to consider the cost of land, the cost of bridges, and the type of soil in the different areas involved. In this section we explain how to incorporate all these factors to build the total cost function. The costs involved in the construction of a highway vary discontinuously with the terrain. Towns and cities, rivers, roads, and mountains appear and must be taken into account. Geographic Information Systems provide the necessary information to estimate the construction costs of a linear unit of road at each point $(x, y)$ of the region in question. Suppose we have a sample of points $\left\{\left(\widehat{x}_{i}, \widehat{y}_{i}\right)\right\}_{i=1}^{n}$ in the study area and that with the help of these GIS we have estimated the construction costs per

linear unit of highway at each point $\widehat{C}_{i}=\widehat{C}\left(\widehat{x}_{i}, \widehat{y}_{i}\right)$ con $i=1, \cdots, n$. These costs will be the sum of the purchase of land, paving, earth movement, building junctions if there are roads and building bridges if there are rivers. Furthermore, the cost function $\widehat{C}\left(\widehat{x}_{i}, \widehat{y}_{i}\right)$, by means of penalty costs, can incorporate information about especial or forbidden areas 
such as national parks, etc.

The problem described is solved by using heuristic algorithms based on assessments of the objective function, which allows the GIS information to be included in the cost assessment. Instead of using the GIS information directly in the model to evaluate the cost function it has been used to construct a piecewise linear function for interpolating the cost function. This has been done to reduce the computational burden. The model uses a bivariate interpolation function of the construction costs, indicated by $C(x, y)$, built from the scattered positional data $\left\{\left(\widehat{x}_{i}, \widehat{y}_{i}, \widehat{C}_{i}\right)\right\}_{i=1}^{n}$ in the following way. Firstly a triangularization of the study region based on the sample has been done (see Figure 3 left). To calculate the cost $C(x, y)$, we consider the vertices $P_{1}, P_{2}, P_{3}$ of the triangle containing the point $(x, y)$. Then we express the point $(x, y)$ as a linear convex combination of the vertices, i.e, $(x, y)=\sum_{i=1}^{3} \beta_{i} P_{i}$ with $\beta_{i} \geq 0$ with $i=1,2,3$ and $\beta_{1}+\beta_{2}+\beta_{3}=1$. Finally, we get $C(x, y)=\sum_{i=1}^{3} \beta_{i} C_{i}$.

In this paper the highway corridor is defined by a segment with endpoints $\left(x_{0}, y_{0}\right)$ and $\left(x_{1}, y_{1}\right)$ (see Figure 3 left). Figure 3 (right hand figure) shows the function $g(\lambda)=$ $C\left((1-\lambda) x_{0}+\lambda x_{1},(1-\lambda) y_{0}+\lambda y_{1}\right)$ with $\lambda \in[0,1]$ which represents the construction cost for each linear unit at each point of the highway. The mean cost of the highway is calculated by the integral $\int_{0}^{1} g(\lambda) \mathrm{d} \lambda$. Finally, we can evaluate the cost of a corridor by multiplying the total length by the mean construction cost of each linear unit, which gives the following expression: 


$$
B\left(x_{0}, y_{0} ; x_{1}, y_{1}\right)=\sqrt{\left(x_{1}-x_{0}\right)^{2}+\left(y_{1}-y_{0}\right)^{2}} \int_{0}^{1} C\left((1-\lambda) x_{0}+\lambda x_{1},(1-\lambda) y_{0}+\lambda y_{1}\right) d \lambda
$$

where $B\left(x_{0}, y_{0} ; x_{1}, y_{1}\right)$ is the corridor cost.

\section{Corridor selection criterion}

The main idea in selecting a corridor is to define what we understand as the best corridor, i.e. in which sense we say that a given corridor is the best possible among all possible selections. In this paper we consider that the best corridor is the one maximizing the served flow given a fixed budget. So, we need to define a function to evaluate the served flow.

In order to determine the flow served by each corridor, we need to know the flow intensities (number of potential users per $\mathrm{Km}^{2}$ ) at each point and their directions, due to each OD pair $i$. To this end, we start by assigning to each OD flow a tubular Gaussian intensity function of the form

$$
f_{i}(x, y)=t_{i} \exp \left[-\frac{d_{i}(x, y)}{2 \sigma_{i}^{2}}\right]
$$

where $t_{i}$ is the corresponding OD pair flow, $d_{i}(x, y)$ is the distance of point $(x, y)$ to the segment which joins the origin and destination of pair $i$, and $\sigma_{i}$ is a distance, associated with the standard deviation of the normal distribution, that measures the width of the tube (see Figure 4). 
The maximum of this function is $t_{i}$, i.e. proportional to the $i$ th OD flow, and is attained all along the segment joining the origin and destination points of this OD pair. This function decreases when the point $(x, y)$ moves apart from this segment, following a Gaussian law with standard deviation $\sigma_{i}$, which means that moving $2 \sigma_{i}$ any side from this segment practically reduces the probability of finding a user of the corresponding OD pair to a very small value $(0.025)$.

Some examples of functions belonging to this family are shown in Figure 4. They refer to the particular examples included in this paper and show the corridors: Ciudad Real-Puertollano, Talavera-Toledo and Albacete-Guadalajara. Since the maxima of these functions are reached at the segment points (wave crest) joining the centers of these cities, if the only flow were that associated with a single pair of cities, the corridors would be located exactly at these segments. However, when several OD flows are considered, the optimal corridors do not exactly align with the straight lines joining cities.

The flow intensity at each point $(x, y)$ of the region is the sum of all the flow intensities generated by all the OD pairs in the study region, that is,

$$
f(x, y)=\sum_{i=1}^{n_{O D}} t_{i} \exp \left[-\frac{d_{i}(x, y)}{2 \sigma_{i}^{2}}\right] .
$$

An example of this function is shown in Figure 5, where the flow intensity can be seen independently of its direction, and where some OD pairs can be easily identified by the almost parallel nature of the level curves, as is the case of the OD pairs Ciudad Real-Puertollano, Talavera-Toledo, Albacete-Guadalajara, Toledo-Ciudad Real, Toledo- 
Albacete, Ciudad Real-Albacete, Guadalajara-Albacete and Guadalajara-Toledo. It is interesting to see how this graph, in spite of dealing with only total flow at each point, still exhibits this directional information.

Thus, the intensity function due to all demands associated with a given corridor $c$ becomes

$$
f_{c}(x, y)=\sum_{i=1}^{n_{O D}} r_{i}(c) t_{i} \exp \left[-\frac{d_{i}(x, y)}{2 \sigma_{i}^{2}}\right],
$$

where $r_{i}(c)$ is a correction factor due to direction, defined in the range $[0,1]$ and $n_{O D}$ is the number of origin-destination pairs.

The proposed model in (4) assumes that the contribution of point $(x, y)$ to the OD $i$ flow served by corridor $c$ depends on two factors: (a) the distance $d_{i}(x, y)$ in Eq. (3) and the degree in which corridor $c$ allows the users of the OD $i$ to reach their destinations in a direct way. The model measures this degree by means of a correction factor $r_{i}(c)$ that is based on the angle between the segment joining the origin and destination of the OD $i$ and the corridor $c$ alignment. The $r_{i}(c)$ function considers that the corridor serves only OD flows whose directions are "close" to the corridor direction, and the fact that the closer the directions the larger the flow proportions served.

In summary, a corridor will serve a given OD pair flow if it is close to the corresponding segment and at the same time both directions are coincident or close. Otherwise, the corridor is not useful to that OD pair.

In the following subsections we analyze the effects of distances $d_{i}(x, y)$ and directions $r_{i}(c)$ separately. 


\section{Effect of distance on the served OD flows}

In this subsection we propose a closed formula for $d_{i}(x, y)$. Let $\left(x_{o}^{i}, y_{o}^{i}\right)$ and $\left(x_{d}^{i}, y_{d}^{i}\right)$ be the coordinates of the origin and the destination of the OD pair $i$. Then, the value of $d_{i}(x, y)$ measures the distance of a given point $(x, y)$ to the segment defined by the origin and destination of pair $i$, and is the minimum of the following quadratic program:

$$
\begin{aligned}
& \text { Minimize } Q=\left(x-x_{o}^{i}(1-\lambda)-x_{d}^{i} \lambda\right)^{2}+\left(y-y_{o}^{i}(1-\lambda)-y_{d}^{i} \lambda\right)^{2} \\
& \lambda
\end{aligned}
$$

subject to:

$$
\begin{gathered}
-\lambda \leq 0 \quad: \quad \mu_{1} \\
\lambda-1 \leq 0 \quad: \quad \mu_{2}
\end{gathered}
$$

The Karush-Khun-Tucker (KKT) optimality conditions (see [Castillo et al., 2002] and [Conejo et al., 2006]) for this problem are:

Gradient condition:

$$
0=2\left(x-x_{o}^{i}(1-\lambda)-x_{d}^{i} \lambda\right)\left(x_{o}^{i}-x_{d}^{i}\right)+2\left(y-y_{o}^{i}(1-\lambda)-y_{d}^{i} \lambda\right)\left(y_{o}^{i}-y_{d}^{i}\right)-\mu_{1}+\mu_{2}
$$

Primal feasible conditions:

$$
\begin{aligned}
-\lambda & \leq 0 \\
\lambda-1 & \leq 0
\end{aligned}
$$

Complementary slackness conditions:

$$
\mu_{1} \lambda=0
$$




$$
\mu_{2}(\lambda-1)=0
$$

Dual feasibility conditions:

$$
\begin{aligned}
& \mu_{1} \geq 0 \\
& \mu_{2} \geq 0 .
\end{aligned}
$$

Let us define

$$
\lambda=-\frac{\left(x-x_{o}^{i}\right)\left(x_{o}^{i}-x_{d}^{i}\right)+\left(y-y_{o}^{i}\right)\left(y_{o}^{i}-y_{d}^{i}\right)}{\left(x_{o}^{i}-x_{d}^{i}\right)^{2}+\left(y_{o}^{i}-y_{d}^{i}\right)^{2}}
$$

The solution to the problem (5)-(7) is achieved in one of the following cases:

Case 1. $\mu_{1}=\mu_{2}=0$. In this case we have $0 \leq \lambda \leq 1$ and

$$
Q_{1}^{*}=\sqrt{\left(x-x_{o}^{i}(1-\lambda)-x_{d}^{i} \lambda\right)^{2}+\left(y-y_{o}^{i}(1-\lambda)-y_{d}^{i} \lambda\right)^{2}} .
$$

Case 2. $\mu_{1}=0 ; \mu_{2} \geq 0 \Rightarrow \lambda=1$. In this case we have:

$$
Q_{2}^{*}=\sqrt{\left(x-x_{d}^{i}\right)^{2}+\left(y-y_{d}^{i}\right)^{2}} .
$$

Case 3. $\mu_{1} \geq 0 ; \mu_{2}=0 \Rightarrow \lambda=0$. In this case we have:

$$
Q_{3}^{*}=\sqrt{\left(x-x_{o}^{i}\right)^{2}+\left(y-y_{o}^{i}\right)^{2}} .
$$

and then, the value $d_{i}(x, y)$ becomes

$$
d_{i}(x, y)=\min \left\{Q_{1}^{*}, Q_{2}^{*}, Q_{3}^{*}\right\}
$$




\section{Effect of direction on the served OD flows}

In order to consider the direction of flow, we have included in formula (4) the function $r_{i}(c)$, defined as

$$
r_{i}(c)= \begin{cases}\cos \left(\alpha \theta_{i c}\right) & \text { if }\left|\theta_{i c}\right| \leq \frac{\pi}{2 \alpha} \\ 0 & \text { otherwise }\end{cases}
$$

where $\alpha$ is a factor that limits the maximum angle capturing flow (see Figure 6), and $\theta_{i c}$ is the angle between the OD segment and the corridor.

If $\left(x_{o}^{i}, y_{o}^{i}\right)$ and $\left(x_{d}^{i}, y_{d}^{i}\right)$ are the endpoints of the OD pair segment, and $\left(x_{0}, y_{0}\right)$ and $\left(x_{1}, y_{1}\right)$ are the endpoints of the corridor, the angle $\theta_{i c}$ is evaluated as

$$
\theta_{i c}= \begin{cases}\tilde{\theta}_{i c} & \text { if } \tilde{\theta}_{i c} \leq \pi /(2 \alpha) \\ 0 & \text { otherwise. }\end{cases}
$$

where $\tilde{\theta}_{i c}=\arccos \left[\frac{\left(x_{d}^{i}-x_{o}^{i}\right)\left(x_{1}-x_{0}\right)+\left(y_{d}^{i}-y_{o}^{i}\right)\left(y_{1}-y_{0}\right)}{\sqrt{\left(\left(x_{d}^{i}-x_{o}^{i}\right)^{2}+\left(y_{d}^{i}-y_{o}^{i}\right)^{2}\right)\left(\left(x_{1}-x_{0}\right)^{2}+\left(y_{1}-y_{0}\right)^{2}\right)}}\right]$.

The value of $r_{i}(c)$ ranges inside the interval $[0,1]$, with $r_{i}(c)=1$ when both directions coincide, and $r_{i}(c)=0$ when the angle formed by both segments is $\theta_{i c} \geq \pi /(2 \alpha)$. This means that the parameter $\alpha$ limits the angle above which the corridor does not serve a given OD pair.

Some examples of the family of functions used to take into account the direction of the corridor for $\alpha=\{1,2,3,4,5,6,7,8,9\}$ are shown in Figure 7 , where it can be seen that this set of values permits a wide range of possibilities. 


\section{The corridor served flow function}

If, as before, $\left(x_{0}, y_{0}\right)$ and $\left(x_{1}, y_{1}\right)$ are the endpoints of the corridor, the function $f_{c}(x, y)$ gives the flow intensity at each point $(x, y)$ of the corridor. The mean flow in the corridor

is given by the integral $\int_{0}^{1} f_{c}\left((1-\lambda) x_{0}+\lambda x_{1},(1-\lambda) y_{0}+\lambda y_{1}\right) d \lambda$, and multiplying by the length of the corridor gives the corridor served flow function:

$$
F\left(x_{0}, y_{0} ; x_{1}, y_{1}\right)=\sqrt{\left(x_{1}-x_{0}\right)^{2}+\left(y_{1}-y_{0}\right)^{2}} \int_{0}^{1} f_{c}\left((1-\lambda) x_{0}+\lambda x_{1},(1-\lambda) y_{0}+\lambda y_{1}\right) d \lambda
$$

where $F\left(x_{0}, y_{0} ; x_{1}, y_{1}\right)$ is the total flow served by the corridor.

\section{The model}

Given the above, our problem can be stated as

$$
\begin{aligned}
& \text { Maximize } F\left(x_{0}, y_{0} ; x_{1}, y_{1}\right) \\
& x_{0}, y_{0} ; x_{1}, y_{1} \\
& \text { subject to: } B\left(x_{0}, y_{0} ; x_{1}, y_{1}\right) \leq B_{0},
\end{aligned}
$$

where $B_{0}$ is the available budget.

\section{Resolution of the corridor problem}

In this Section we analyze the characteristics of the proposed problem and consider several heuristic methods for solving it. 


\section{Reformulation of the model}

The computational methods available to solve the proposed problem (23)-(24) may produce instability when the search leaves the domain of the cost function $B\left(x_{0}, y_{0} ; x_{1}, y_{1}\right)$. We now set out the procedure for avoiding this phenomenon. We first limit the space in which the corridor may be located to the rectangle $\mathcal{R}$ defined by the endpoints $\left(x_{\min }, y_{\min }\right)$, $\left(x_{\min }, y_{\max }\right),\left(x_{\max }, y_{\min }\right)$ and $\left(x_{\max }, y_{\max }\right)$, the endpoints being defined by:

$$
x_{\text {min }}=\min _{c \in \mathcal{O}} x_{c}, \quad x_{\max }=\max _{c \in \mathcal{O}} x_{c}, \quad y_{\min }=\min _{c \in \mathcal{O}} y_{c}, \quad y_{\max }=\max _{c \in \mathcal{O}} y_{c}
$$

where $\mathcal{O}$ is the set of cities, and $\left(x_{c}, y_{c}\right)$ their UTM-coordinates. We assume that the corners of the rectangle are included in the sampling $\left\{\left(\widehat{x}_{i}, \widehat{y}_{i}\right)\right\}_{i=1}^{n}$ of the cost function with values that penalise their location. In this way we can define the cost function $B\left(x_{0}, y_{0} ; x_{1}, y_{1}\right)$ over the rectangle $\mathcal{R}$ which contains the study region. This new constraint, that the corridor be contained in the rectangle $\mathcal{R}$, does not affect the optimal solution to the problem since the space eliminated does not attract additional demand.

The next step is to transform the initial problem into an unconstrained optimization problem. This is necessary because the function of $B\left(x_{0}, y_{0} ; x_{1}, y_{1}\right)$ is complicated to handle mathematically. For this reason we penalise the budget constraint in the ob-

jective function and make use of a biunivocal transformation of $\mathbb{R}^{2}$ on the rectangle $\mathcal{R}=\left[x_{\min }, x_{\text {max }}\right] \times\left[y_{\min }, y_{\max }\right]$ in order to eliminate the constraint that the corridor must be contained in the rectangle $\mathcal{R}$. 
To this end, we can use the following change of variables

$$
\begin{aligned}
& x_{i}=g\left(u_{i}\right)=x_{\text {min }}+\left(x_{\text {max }}-x_{\text {min }}\right)\left(\frac{\arctan u_{i}}{\pi}+1 / 2\right) \\
& y_{i}=h\left(v_{i}\right)=y_{\text {min }}+\left(y_{\text {max }}-y_{\text {min }}\right)\left(\frac{\arctan v_{i}}{\pi}+1 / 2\right)
\end{aligned}
$$

and its inverse

$$
\begin{aligned}
& u_{i}=g^{-1}(x)=\tan \left[\pi\left(\frac{x_{i}-x_{\min }}{x_{\max }-x_{\min }}-1 / 2\right)\right] \\
& v_{i}=h^{-1}(v)=\tan \left[\pi\left(\frac{y_{i}-y_{\min }}{y_{\max }-y_{\min }}-1 / 2\right)\right],
\end{aligned}
$$

where the new variables $u_{i}$ and $v_{i}$ are unrestricted, while $x_{\min } \leq x_{i} \leq x_{\max }$ and $y_{\min } \leq$ $y_{i} \leq y_{\max }$

With this change of variable and using a high weight value $\rho>0$, our optimization problem (23)-(24) can be stated as

$$
\text { Maximize } F\left(g\left(u_{0}\right), h\left(v_{0}\right) ; g\left(u_{1}\right), h\left(v_{1}\right)\right)-\rho \max \left\{0,\left(B\left(g\left(u_{0}\right), h\left(v_{0}\right) ; g\left(u_{1}\right), h\left(v_{1}\right)\right)-B_{0}\right)\right\}^{2},
$$
$u_{0}, v_{0} ; u_{1}, v_{1}$

and once solved we calculate $x_{0}, y_{0}, x_{1}$ and $y_{1}$ using (25)-(26) to return to the initial coordinates.

\section{Resolution methods}

Corridor selection is a multi-objective decision problem in which it is necessary to evaluate not just the demand to be satisfied but also other environmental and social benefits. The method used should, therefore, locate, as well as the optimal corridor with regard to the 
demand to be served, a set of corridors which reach a satisfactory level of demand. These corridors coincide with global and local minima of the proposed optimization model. To illustrate this matter consider Figure 8. It shows several local optima of the real problem used as a case study in the numerical trials. These figures represent various corridors present in the study zone, each serving different sets of origin-destination pairs. These local minima satisfy the condition that small changes in the location de not improve the objective function. The solution method should identify these corridors and locate them optimally in the area.

Due to the nature of the problem, the proposed solution method consists of generating local optima by means of a heuristic algorithm and choosing those solutions which are no more than a given percentage away from the best solutions found. The choice of a heuristic algorithm to obtain the local optima should be based on the following characteristics of the model:

- Unconstrained nonlinear optimization and using derivative-free. Problem (29) is an unconstrained nonlinear optimization model. Obtaining closed formulae for the derivatives of functions $F\left(g\left(u_{0}\right), h\left(v_{0}\right) ; g\left(u_{1}\right), h\left(v_{1}\right)\right)$ and $B\left(g\left(u_{0}\right), h\left(v_{0}\right) ; g\left(u_{1}\right), h\left(v_{1}\right)\right)$ is a complicated task, or they do not exist and so a choice should be made from among the unconstrained nonlinear optimization derivative-free methods.

- Computational cost. The computational cost of assessing the budget constraint $B\left(g\left(u_{0}\right), h\left(v_{0}\right) ; g\left(u_{1}\right), h\left(v_{1}\right)\right)$ is high as it is based on evaluating the two-variable 
interpolation function, and this should be borne in mind. This means that the speed of convergence is an important parameter in the choice of an heuristic algorithmn.

With regard to these considerations, in this paper we have worked with four heuristic algorithms.

- Simulated Annealing method (SA, [Kirpatrick et al., 1983]). This algorithm has properties of global convergence which allows the generation of an optimal corridor with respect to the corridor served flow function (23). The SA is a probabilistic search method which allows the process to be repeated to generate multiple local optima.

- Modified Simulated Annealing method (MSA, [Vanderbilt and Louie, 1984]). This algorithm introduces an improvement of the SA algorithm which involves obtaining search directions by taking into account the progress achieved in the previous stages. This modification accelerates the convergence of the SA as has been reported in the works of ([Friesz et al., 1992] and [García and Marín, 2002]).

- Particle Swarm Optimization. (PSO, [Kennedy and Eberhart, 1995]). This method is now being applied in many fields with considerable success. The reason for choosing this algorithm is that it is a very robust method for finding the global optimum of an optimization problem.

- Nelder-Mead method (NM, [Nelder and Mead, 1965]). This method has very pronounced local convergence. The reason for working with this method is to be able 
to generate multiple local optima.

The SA, MSA and NM algorithms start from an initial corridor while the PSO starts from a set of initial solutions. The convergence of these methods depends on the initialization. In this paper we use the SA, MSA and NM methods with ten different initializations and choose the best solution generated. The PSO algorithm uses as its initial set the ten previous initializations and will be run just once. The way in which these initializations are generated is a key task in the overall behaviour of the algorithm. For this reason we have analyzed three different ways of generating the initial set.

- Uniform distribution. The initial corridors are generated in such a way that they are uniformly distributed throughout the feasible region (rectangle $\mathcal{R}$ ).

- Random. The initial corridors are generated randomly by a uniform distribution in the rectangle $\mathcal{R}$.

- Heuristic Method. We have designed a process in which we first evaluate the importance of each city with respect to the travel flow, which is measured as the sum of the input and output flows associated with each city, so that

$$
\mathcal{I}_{i}=\sum_{i=1}^{n} \sum_{j=1}^{n}\left(O D_{i, j}+O D_{j, i}\right),
$$

where $\mathcal{I}_{i}$ is the importance of city $i$ and $O D_{i, j}$ is the number of users traveling from origin $i$ to destination $j$. 
We need to select the best solution, that is, the one maximizing the total flow subject to the budget constraint. To this end, we proceed using the algorithm given in Figure 9.

\section{Numerical experiments}

\section{Description of the test problem}

The region of Castilla-La Mancha (Spain) has an area of 79, 226 square kilometres. We have considered the 67 towns with the highest populations and worked with the demands among these towns, as estimated by the INE (National Institute of Statistics). In the study problem 1,626 Origin-Destination pairs have been considered, using the objective function defined in Eq. (22) and two cost functions $B$ have been analysed:

$\left(B_{1}\right)$ : Interpolation formula. The model used is set out in Subsection 2.1, where a linear interpolation function is formed from the input data. In this case the input data has $n=9718$ points which represent the construction costs in hundreds of millions of euros (because of rivers, mountains, soil types, etc.) for the region of Castilla-La Mancha. Firstly a triangularization of the study region is carried out, generating 19325 triangles as shown in Figure 10. From these data the piecewise linear interpolation function is obtained via the expression (1) of Subsection 2.1. Let this function be called $B_{1}$. 
$\left(B_{2}\right)$ : The land cost. We now consider a second test problem consisting of the same OD matrix as the previous case, that is the users wish to travel as before, but different construction costs are assumed.

The land cost is an important part of the total cost of highways and corridors, due to its relative importance with respect to the cost of infrastructure and other costs. Thus, the important differences between rural and urban areas play a decisive role when deciding between different possible new corridor or highway locations. To insert a highway inside a city is on the one hand highly convenient, because it facilitates user access to the city centers, but on the other hand it has very high associated costs, so that a trade-off is required. On the other hand, the cost of land in rural areas is low. This justifies and explains the tendency to move important highways sufficiently far from the city in order to attain an affordable total cost. Consequently, the cost of land is one of the main ingredients that cannot be ignored in a corridor location selection process.

In order to consider the increase of land cost with the distance to the center of the cities, we use a model that takes this into account. We use a sum of Gaussian kernels for each city of the type

$$
C_{2}(x, y)=C_{1}+\sum_{j=1}^{n} C_{\max }^{j} \exp \left[-\frac{\left(x-x_{j}^{0}\right)^{2}+\left(y-y_{j}^{0}\right)^{2}}{2 \sigma_{j}^{2}}\right]
$$

where $n$ is the number of cities, $C_{\max }^{j}$ is the land cost at the city center, $\left(x_{j}^{0}, y_{j}^{0}\right) ; j=$ $1,2, \ldots, n$ are the city center coordinates, $C_{1}$ is a constant of the construction whose 
value in all trials is 0.002 and $\sigma_{j}$ is the standard deviation value, which represents half of the distance where city $j$ has some relevant influence on the land cost.

Finally, the cost $B_{2}$ of a corridor with co-ordinates $\left(x_{0}, y_{0}\right)$ and $\left(x_{1}, y_{1}\right)$ is given by: $B_{2}\left(x_{0}, y_{0} ; x_{1}, y_{1}\right)=\sqrt{\left(x_{1}-x_{0}\right)^{2}+\left(y_{1}-y_{0}\right)^{2}} \int_{0}^{1} C_{2}\left((1-\lambda) x_{0}+\lambda x_{1},(1-\lambda) y_{0}+\lambda y_{1}\right) d \lambda$,

where $B_{2}\left(x_{0}, y_{0} ; x_{1}, y_{1}\right)$ is the corridor cost.

To illustrate, we present in Figure 11 (left hand figure) the resulting land cost function for the case of the Castilla-La Mancha region, where the cost of land in the cities is shown to be related to the city sizes. The corresponding level curves of this function are shown in Figure 11 (right hand figure).

As we shall see later, this cost function produces a displacement of the optimal corridor locations outside the city centers. In other words, optimal cost corridors tend to move outwards from the city centers, but not too much in order to serve more users during their longest possible journey distances.

\section{Comparative of heuristics}

The SA, MSA and, PSO and NM algorithms were codified in MATLAB ([MathWorks, 2004]) and run on a Pentium computer with a $2.66 \mathrm{GHz}$ Intel (R) microprocessor Core(TM) 2 Quad CPU Q9450 with 4 GB of RAM. The penalty parameter $\rho$ of problem (29) was taken as $\rho=10000$ in all trials. The SA and MSA algorithms use the parameters $\alpha$ (maximum 
length of movement) and $\beta$ (cooling parameter). The values $\alpha=0.04$ and $\beta=-2.75$ were used (see [Kirpatrick et al., 1983]). The MSA algorithm has an additional parameter $M$ which shows the number of correct solutions after which the Choleski method is used to update the direction generation method (see [Vanderbilt and Louie, 1984]). In the numerical trials the value $M=20$ was taken.

The computational results obtained are shown in Tables 2 and 3. These tables show the value obtained for $F$, the corridor cost, and the CPU time used (measured in seconds). All these data are shown for the three initializations, for all the algorithms and for the two cost functions $B_{1}$ and $B_{2}$.

It can be seen that working with the interpolation function $B_{1}$ increases the CPU time by $50-100$ times that required for an analytic function $B_{2}$. In spite of this, however, the results show that the computational costs of all the heuristics are acceptable for this type of problem and therefore they allow the flexibility of $B_{1}$ to be used to include costs.

With respect to the initialization it is seen that the uniform initialization gives the best results, as, if a global optimization is desired it is better to complete explore the entire feasible solution space. Heuristic initialization tends to locate local optima while random initialization does not consider the whole region.

To understand better the behaviour of the algorithms, Figure 12 shows the best solution obtained with each of the algorithms for cost $B_{1}$ (left figure in Figure 12) and for cost $B_{2}$ (right figure in Figure 12). For the problem associated with $B_{1}$ there are two corridors with very similar values of the objective function. The MSA and SA algorithms converge 
to one of these solutions and the PSO and NM algorithms to the other. For problem $B_{2}$ the NM gets stuck in a local optimum. The results given by the PSO algorithm are not as expected for cost $B_{2}$, since they don't improve those given by SA and MSA. It can be used to identify the corridor but not to locate it optimally. We have confirmed that this is due to the number of initial particles, 10 being insufficient. Several additional trials have been carried out with initial particle numbers $n=20,30,40,50,60,70,90$ and 100 and it is found that for values higher than $n=30$ it locates the corridor optimally, in a CPU time in the range $200-1000$ seconds.

The results suggest that the MSA algorithm improves the SA algorithm with respect to the computational cost $C P U$ and the objective function, which was to be expected based on previous studies including [García and Marín, 2002]. The results obtained with the NM algorithm for this problem are not satisfactory as they converge to local optima.

\section{A case study}

The location of a corridor is a multi-objective decision problem which depends on hard factors like demand to be serviced and construction costs and other soft factors. The model described is a tool which allows the corridors which attract most demand for a given budget to be identified automatically. From this candidate set the final choice of a corridor will be made after assessing the candidates with regard to the soft criteria. In this section we illustrate how the model is used taking a case study in the region of Castilla-La Mancha (Spain) (see Figure 10). 
The objective sought is to identify a set of good corridors with respect to demand satisfied. The method used to generate this set was to solve the model ten times and to select those corridors which are at most $10 \%$ worse than the best corridor found. The numerical experiment in Subsection 4.2 shows that the MSA algorithm, together with the uniform initialization is a good strategy for solving the problem. We have solved the problem for the cost functions $B_{1}$ and $B_{2}$ and for three different budgets. The results are shown in Figure 13. In the first and second columns the results are given respectively for the cost functions $B_{1}$ and $B_{2}$. The best corridor is shown as a continuous segment while the other good corridors appear as dashed segments.

These results show the effect of the budget on the location of the corridor. An increase in the budget does not only affects the number of kilometers it is possible to build, and the location of the endpoints but also in the placing of the corridor itself. Depending on the available budget the model identifies one corridor or another.

Figure 14 shows the three corridors found and the flow intensity through them (Right hand figure). These three alternatives need to pass a further decision process, based on criteria different from the attraction of demand, which determines the final corridor choice.

The continuous location model determines the endpoints of the corridor and the potential demand for a given budget. These output data are used in the next stage of Highway Alignment Optimization to determine highway alignment. For illustrative purposes, the existing highways in the region of Castilla-La Mancha have been added in Figure 14 (Left 
hand figure). These highways may be considered as examples of the ouput at the HAO stage.

Figure 15 shows the flow captured by each of the corridors seen in Figure 14 for each value of $\lambda$.

\section{Conclusions}

The location of highway corridors obeys certain hard factors, like demand and construction costs, and soft factors. It is crucial to have analytical tools to generate a set of alternatives which can be assessed with respect to all these criteria. In this paper a continuous location model is described which automatically generates a set of optimal corridors with the aim of maximizing the attraction of demand. The model, when considering the performance of a given corridor, takes into account the direction and distance with respect to all the origin-destination pairs in the study region. Furthermore, the model works with an interpolation based cost function which makes it highly flexible and allows a very accurate consideration of many factors involved in the cost of a highway such as the purchase of land, earth movement, location of bridges and intersections etc.

The output from the model is aimed at producing the inputs necessary for the HAO stage such as the endpoints of the alignment and the demand served. This paper, therefore, complements a great deal of other work already existing in the literature.

Four heuristic solution methods have been used: PSO, SA, MSA and NM, in order 
to find a quasi-optimal solution to the model. The nature of the problem means that as well as searching for global optima of the optimization model it is also necessary to find the best local optima. The numerical results suggest that the MSA is the most suitable for this feature of the problem, since it is able to save local minima with low demand attraction and arrive at better solutions with a reduced computational cost.

The methodology has been tested on a case study in the region of Castilla-La Mancha (Spain); it has been shown to be acceptable and the results have been compared and contrasted.

\section{Acknowledgements}

The research carried out and described here is financed by the following projects: PT2007-003-08CCPP del Ministerio de Fomento [Ministry of Development] (CEDEX) and PCC08-0081-4388-2 of the Junta de Comunidades [Autonomous Government] of CastillaLa Mancha (Spain).

\section{References}

[Castillo et al., 2002] Castillo, E., Conejo, A., Pedregal, P., García, R., and Alguacil, N. (2002). Building and Solving Mathematical Programming Models in Engineering and Science. Pure and Applied Series (PAMS), John Wiley \& Sons, Inc., New York. 
[Cheng and Lee, 2006] Cheng, J. F. and Lee, Y. (2006). Model for three-dimensional highway alignment. ASCE Journal of Transportation Engineering, 132(12):913-920.

[Chew et al., 1989] Chew, E. P., Goh, C. J., and Fwa, T. F. (1989). Simultaneous optimization of horizontal and vertical alignments for highways. Transportation Research Part B, 23(5):315-329.

[Conejo et al., 2006] Conejo, A., Castillo, E., Mínguez, R., and García-Bertrand, R. (2006). Decomposition Techniques in Mathematical Programming: Engineering and Science Applications. Springer, Berlin, Heildelberg.

[Drezner and Wesolowsky, 2003] Drezner, Z. and Wesolowsky, G. O. (2003). Network design: Selection and design of links and facility location. Transportation Research A, $37(3): 241-256$.

[Easa, 1988] Easa, S. M. (1988). Selection of roadway grades that minimize earthwork cost using linear programming. Transportation Research, Part A, 22(2):121-136.

[Easa, 1999] Easa, S. M. (1999). Optimum vertical curves for highway profiles. Journal of Surveying Engineering, 125(3):147-157.

[Easa and Mehmood, 2007] Easa, S. M. and Mehmood, A. (2007). Establishing highway horizontal alignment to maximize design consistency. Canadian Journal of Civil Engineering, 34(9):1159-1168. 
[Friesz et al., 1992] Friesz, T. L., Cho, H.-J., Tobin, N., and Anandalingam, G. (1992). A simulated annealing approach to the network design problem with variational inequality constraints. Transportation Science, 26:18-26.

[Fwa, 1989] Fwa, T. F. (1989). Highway vertical alignment analysis by dynamic programming. Transportation Research Record, 1239:1-9.

[Fwa et al., 2002] Fwa, T. F., Chan, W. T., and Sim, Y. P. (2002). Optimal vertical alignment analysis for highway design. ASCE Journal of Transportation Engineering, 128(5):395-402.

[García and Marín, 2002] García, R. and Marín, A. (2002). Parking capacity and pricing in park'n ride trips: A continuous equilibrium network design problem. Annals of Operations Research, 116:153-178.

[Göktepe et al., 2009] Göktepe, A. B., Altun, S., and Ahmedzade, P. (2009). Optimization of vertical alignment of highways utilizing discrete dynamic programming and weighted ground line. Turkish Journal of Engineering and Environmental Sciences, $33(2): 105-116$.

[Jha, 2001] Jha, M. K. (2001). Using a GIS for automated decision-making in highway cost analysis. Transportation Research Record, 1768:260-267.

[Jha, 2003] Jha, M. K. (2003). Criteria-based decision support system for selecting highway alignments. Journal of Transportation Engineering, 129:33-41. 
[Jha and Kim, 2004] Jha, M. K. and Kim, E. (2004). Demand considerations in locating transportation facilities: A case of highway alignments. Advances in Transport, 16:131140.

[Jha et al., 2001] Jha, M. K., McCall, C., and Schonfeld, P. (2001). Using GIS, Genetic Algorithms, and Visualization in highway development. Computer-Aided Civil and Infrastructure Engineering, 16(6):399-414.

[Jha and Schonfeld, 2000a] Jha, M. K. and Schonfeld, P. (2000a). Geographic information system-based analysis of right-of-way cost for highway optimization. Transportation Research Record, 1719:241-249.

[Jha and Schonfeld, 2000b] Jha, M. K. and Schonfeld, P. (2000b). Integrating genetic algorithms and geographic information system to optimize highway alignments. Transportation Research Record, 1719:233-240.

[Jha and Schonfeld, 2003] Jha, M. K. and Schonfeld, P. (2003). Trade-offs between initial and maintenance costs of highways in cross-slopes. Journal of Infrastructure Systems, $9(1): 16-25$.

[Jha and Schonfeld, 2004] Jha, M. K. and Schonfeld, P. (2004). A highway alignment optimization model using geographic information systems. Transportation Research Part A, 38:455-481. 
[Jong and Schonfeld, 2003] Jong, J. C. and Schonfeld, P. (2003). An evolutionary model for simultaneously optimizing threedimensional highway alignments. Transportation Research Part B, 37:107-128.

[Kang, 2008] Kang, M. W. (2008). An Alignment Optimization Model for A Simple Highway Network. PhD thesis, College Park, Md., University of Maryland.

[Kang et al., 2009] Kang, M. W., Schonfeld, P., and Yang, N. (2009). Computer-aided civil and infrastructure engineering. Journal of Advanced Transportation, 24(2):109119.

[Kennedy and Eberhart, 1995] Kennedy, J. and Eberhart, R. (1995). Particle swarm optimization. IEEE International Conference on Neural Networks - Conference Proceedings, 4:1942-1948.

[Kim et al., 2008] Kim, B. J., Kim, W., and Song, B. H. (2008). Sequencing and scheduling highway network expansion using a discrete network design model. Annals of Regional Science, 42(3):621-642.

[Kim et al., 2004a] Kim, E., Jha, M. K., Lovell, D. J., and Schonfeld, P. (2004a). Intersection modeling for highway alignment optimization. Computer-Aided Civil and Infrastructure Engineering, 19(2):119-129. 
[Kim et al., 2004b] Kim, E., Jha, M. K., and Schonfeld, P. (2004b). Intersection construction cost functions for alignment optimization. Journal of Transportation Engineering, 130(2):194-203.

[Kim et al., 2005] Kim, E., Jha, M. K., and Son, B. (2005). Improving the computational efficiency of highway alignment optimization models trough a stepwise genetic algorithms approach. Transportation Research Part B, 39:339-360.

[Kirpatrick et al., 1983] Kirpatrick, S., Gelatt, C. D., and Vechi, M. P. (1983). Optimization by simulated annealing. Science, 220(4598):671-680.

[Lee and Cheng, 2001] Lee, Y. and Cheng, J.-F. (2001). Optimizing highway grades to minimize cost and maintain traffic speed. Journal of Transportation Engineering, 127(4):303-310.

[Maji and Jha, 2009] Maji, A. and Jha, M. K. (2009). Multi-objective highway alignment optimization using a genetic algorithm. Journal of Advanced Transportation, 43(4):481504.

[MathWorks, 2004] MathWorks (2004). Using Matlab Graphics. Matlab Programming. Matlab Desktop Tools and Development Environment. Version \%. MathWorks Inc.

[Miao et al., 2009] Miao, K., Li, L., Yang, X.-L., and Huo, Y.-Y. (2009). Ant colony optimization algorithm for vertical alignment of highways. Geotechnical Special Publication, 189:99-108. 
[Nelder and Mead, 1965] Nelder, J. A. and Mead, R. (1965). A simplex method for function minimization. Computer Journal, 7:308-313.

[Parker, 1977] Parker, N. A. (1977). Rural highway route corridor selection. Transportation Planning and Technology, 3:247-256.

[Petersen, 2002] Petersen, E. R. (2002). A highway corridor planning model: QROAD. Transportation Research Part A: Policy and Practice, 36(2):107-125.

[Solanki et al., 1998] Solanki, R. S., Gorti, J. K., and Southworth, F. (1998). Using decomposition in large-scale highway network design with a quasi-optimization heuristic. Transportation Research Part B: Methodological, 33(12):127-140.

[Trietsch, 1987a] Trietsch, D. (1987a). Comprehensive design of highway networks. Transportation Science, 21(1):26-35.

[Trietsch, 1987b] Trietsch, D. (1987b). A family of methods for preliminary highway alignment. Transportation Science, 21(1):17-25.

[Turner and Miles, 1971] Turner, A. K. and Miles, R. D. (1971). A computer assisted method of regional route location. Highway Research Record, 348:1-15.

[Vanderbilt and Louie, 1984] Vanderbilt, D. and Louie, S. G. (1984). A Monte Carlo Simulated Annealing Approach to Optimization over Continuous Variables. J. Comp. Phys., 56:259-271. 
[Zhu et al., 2010] Zhu, N., Wei, L., Shao, C., and B., J. (2010). The national highway network layout optimization based on combination optimization model. Jiaotong Yunshu Xitong Gongcheng Yu Xinxi/Journal of Transportation Systems Engineering and Information Technology, 10(4):118-124. 


\section{Figure captions list}

1. Stages in highway design and planning.

2. Representation of a corridor.

3. Cost function for a highway.

4. Examples of Gaussian demand functions for a pair of cities.

5. Flow intensity independently of its direction with function $f(x, y)(3)$.

6. Effect of direction on the served OD flows.

7. Some examples of the family of functions $r_{i}(c)$ used to take into account the direction of the corridorr.

8. Local optimal solution.

9. Heuristic initialization.

10. Triangularization of the study region for $B_{1}$.

11. Cost function showing the influence and level curves of the urban and town areas.

12. Best solutions obtained with each heuristic algorithm for cost $B_{1}$ and for cost $B_{2}$.

13. Best solutions with different budgets for cost functions $B_{1}$ and $B_{2}$.

14. Corridors found. 
15. Flow in the corridors found. 


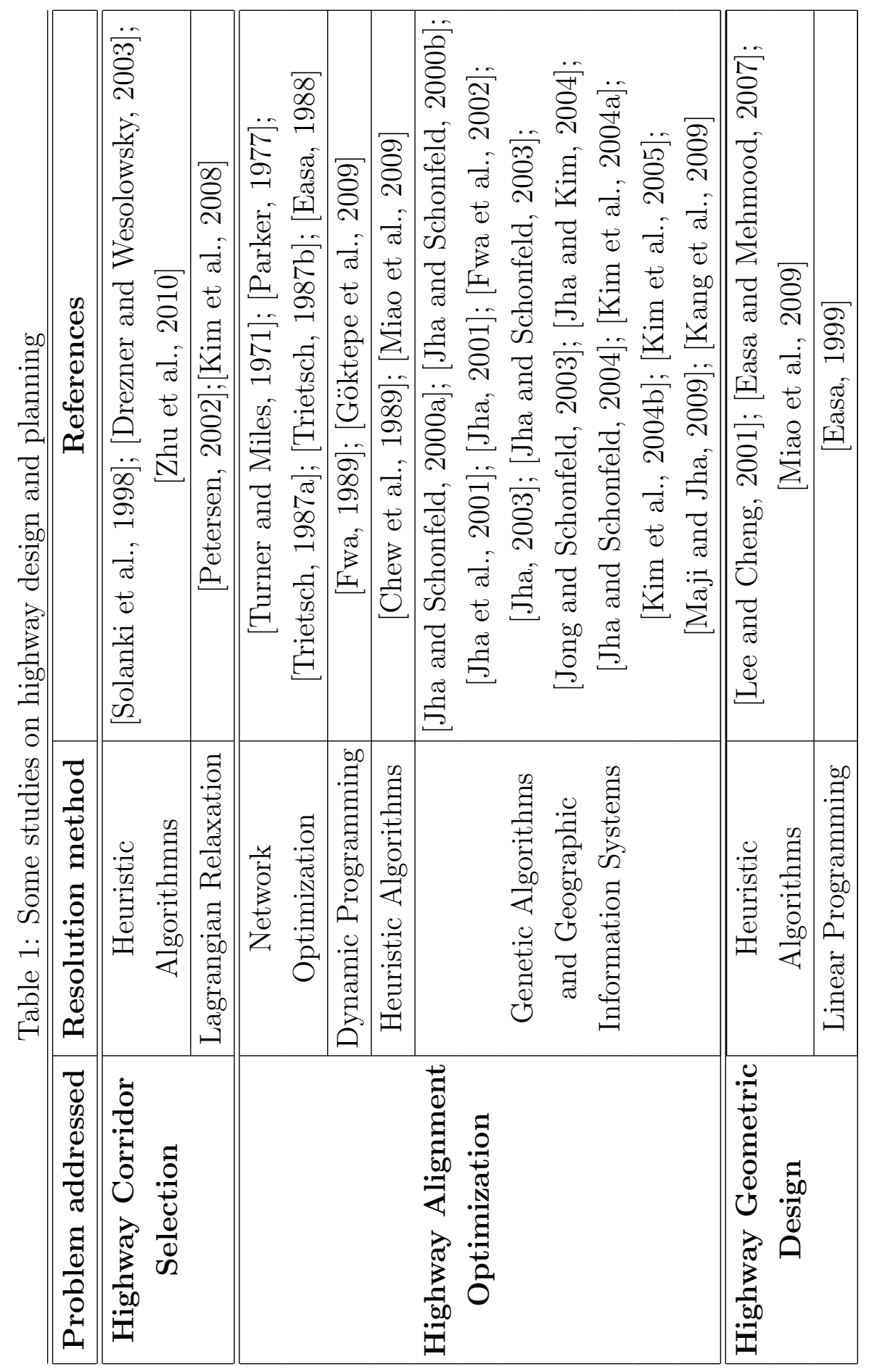


Table 2: Results with cost $B_{1}$

\begin{tabular}{|c|c|c|c|c|c|c|c|c|c|}
\hline \multirow{2}{*}{ Heuristic } & \multicolumn{2}{|c|}{ Uniform Initialization } & \multicolumn{2}{c|}{ Random Initialization } & \multicolumn{3}{c|}{ Heuristic Initialization } \\
\cline { 2 - 10 } & $F$ & $B_{1}$ & $C P U($ sec. $)$ & $F$ & $B_{1}$ & $C P U($ sec. $)$ & $F$ & $B_{1}$ & $C P U($ sec. $)$ \\
\hline SA & 1318.7 & 0.2499 & 7165 & 1115.0 & 0.2498 & 7357 & 1277.6 & 0.2499 & 7310 \\
\hline MSA & 1396.8 & 0.2517 & 6449 & 1355.9 & 0.2531 & 5618 & 1446.7 & 0.2530 & 5709 \\
\hline PSO & 1451.2 & 0.2509 & 4368 & 1016.5 & 0.2508 & 3295 & 976.3 & 0.2501 & 3271 \\
\hline NM & 453.7 & 0.2486 & 4416 & 294.9 & 0.2490 & 3294 & 1289.0 & 0.2500 & 5308 \\
\hline
\end{tabular}

Table 3: Results with cost $B_{2}$

\begin{tabular}{|c|c|c|c|c|c|c|c|c|c|}
\hline \multirow{2}{*}{ Heuristic } & \multicolumn{3}{|c|}{ Uniform Initialization } & \multicolumn{3}{c|}{ Random Initialization } & \multicolumn{3}{c|}{ Heuristic Initialization } \\
\cline { 2 - 10 } & $F$ & $B_{2}$ & $C P U($ sec. $)$ & $F$ & $B_{2}$ & $C P U($ sec. $)$ & $F$ & $B_{2}$ & $C P U($ sec. $)$ \\
\hline SA & 1049.4 & 0.6008 & 143 & 1001.9 & 0.6006 & 143 & 751.7 & 0.6013 & 172 \\
\hline MSA & 1110.4 & 0.5941 & 132 & 1132.6 & 0.6050 & 132 & 911.1 & 0.5973 & 142 \\
\hline PSO & 865.8 & 0.6008 & 100 & 785.5 & 0.6005 & 74 & 775.4 & 0.6004 & 76 \\
\hline NM & 584.3 & 0.6000 & 23 & 531.6 & 0.5999 & 28 & 683.9 & 0.6000 & 37 \\
\hline
\end{tabular}




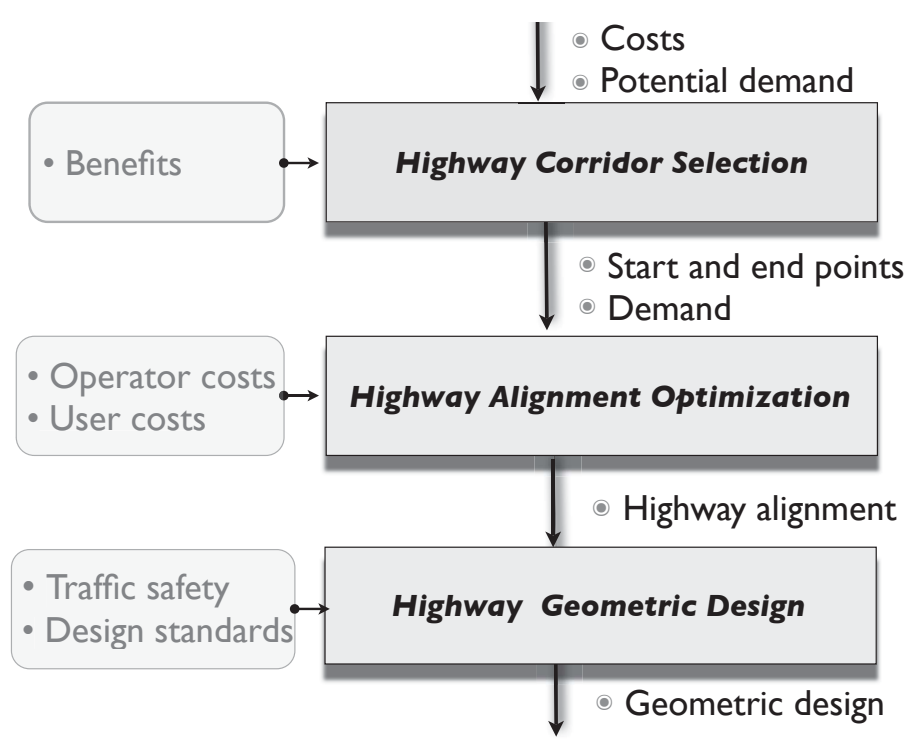

Figure 1: Stages in highway design and planning

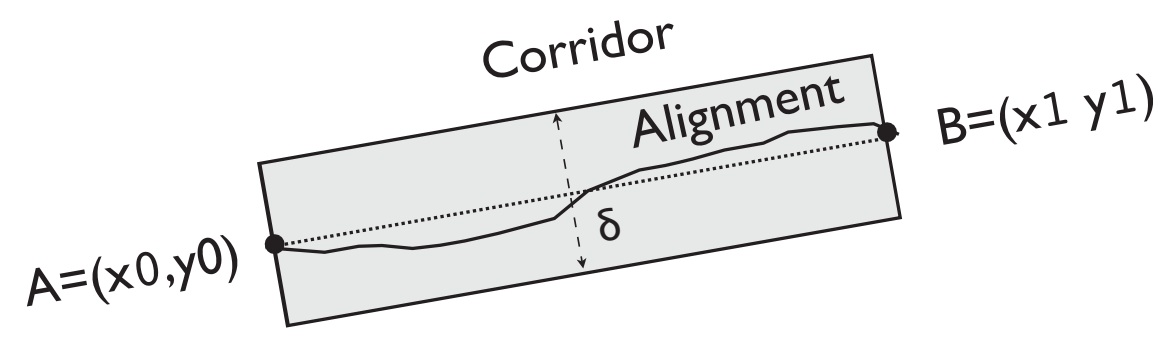

Figure 2: Representation of a corridor 

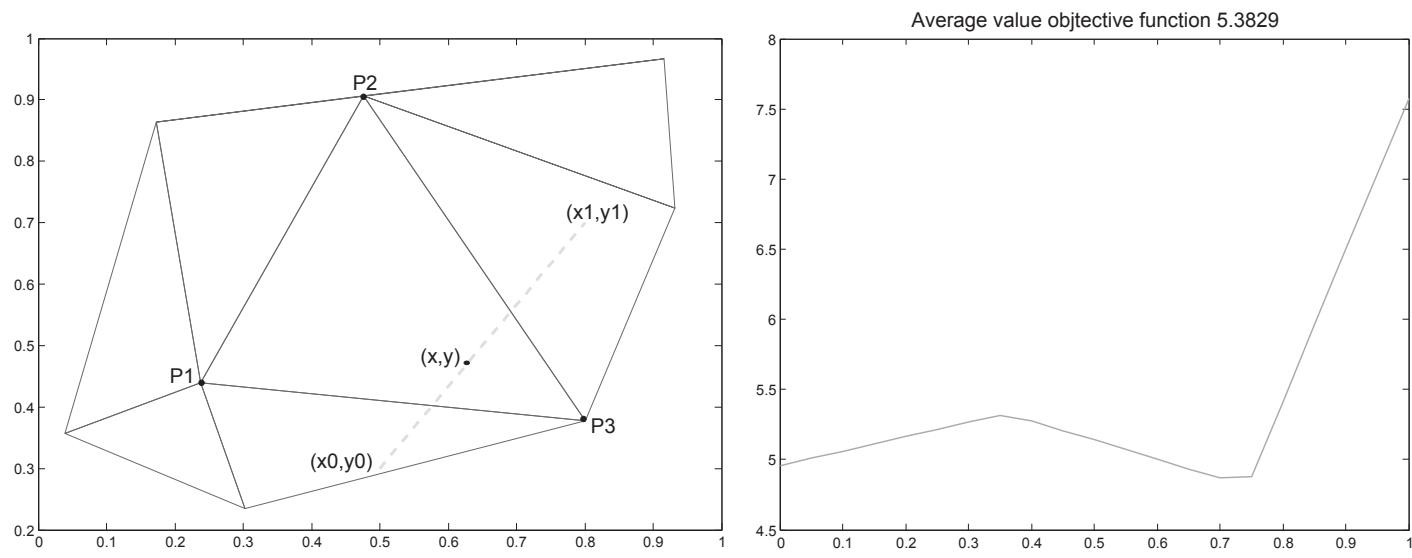

Figure 3: Cost function for a highway

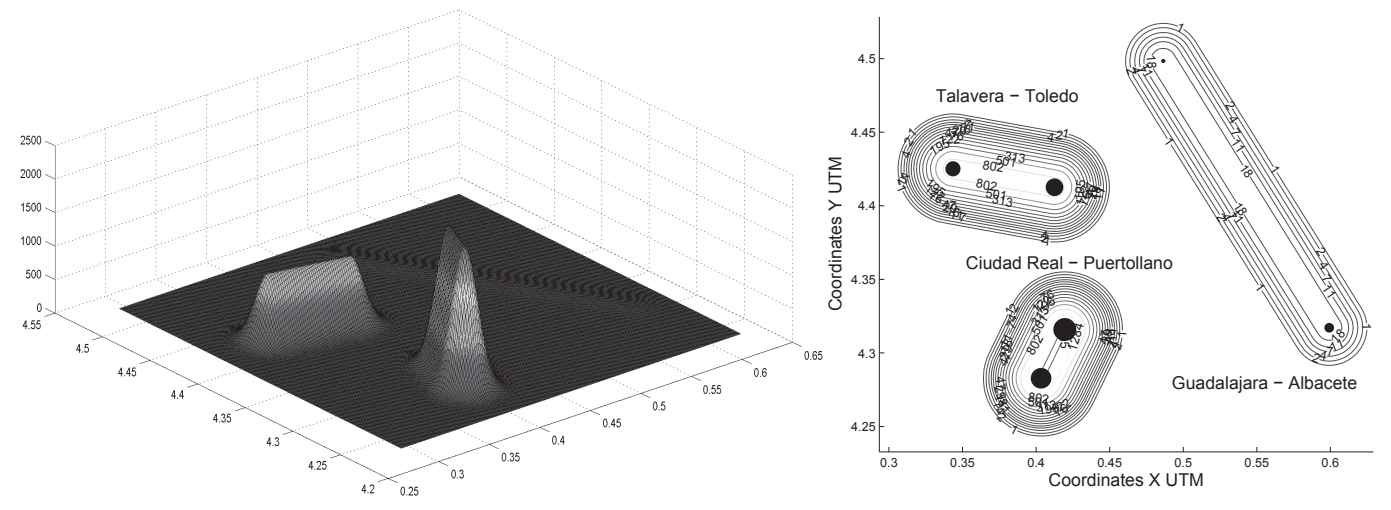

Figure 4: Examples of Gaussian demand functions for a pair of cities 

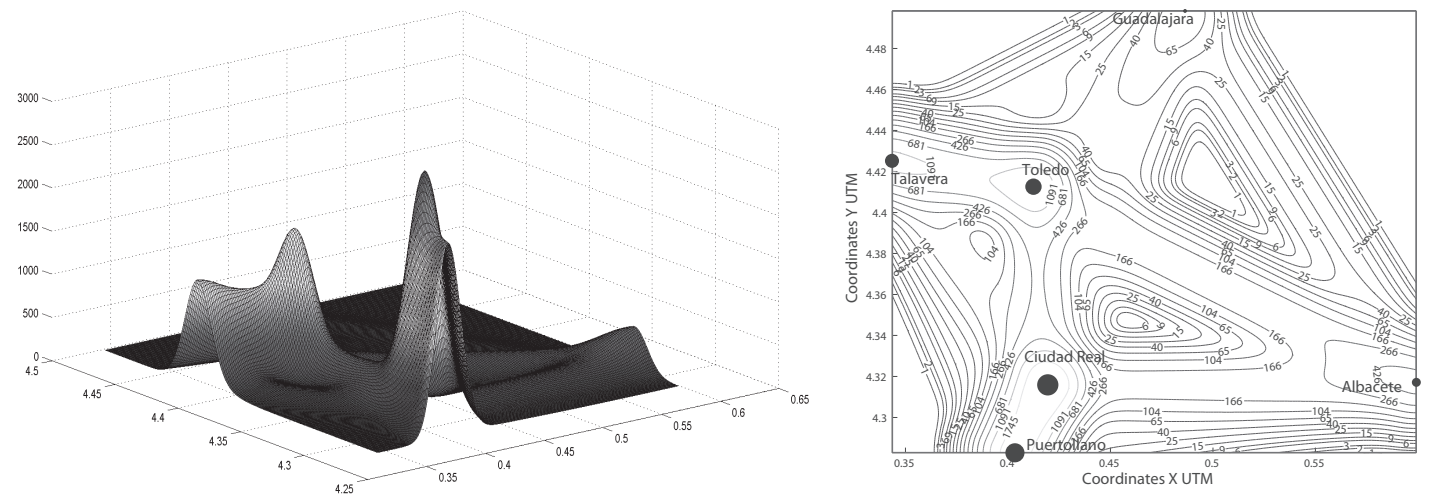

Figure 5: Flow intensity independently of its direction with function $f(x, y)(3)$

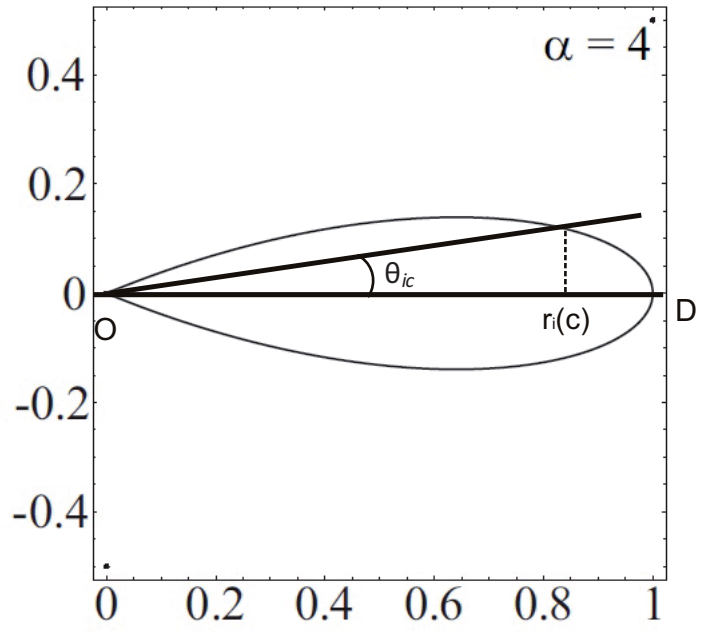

Figure 6: Effect of direction on the served OD flows 

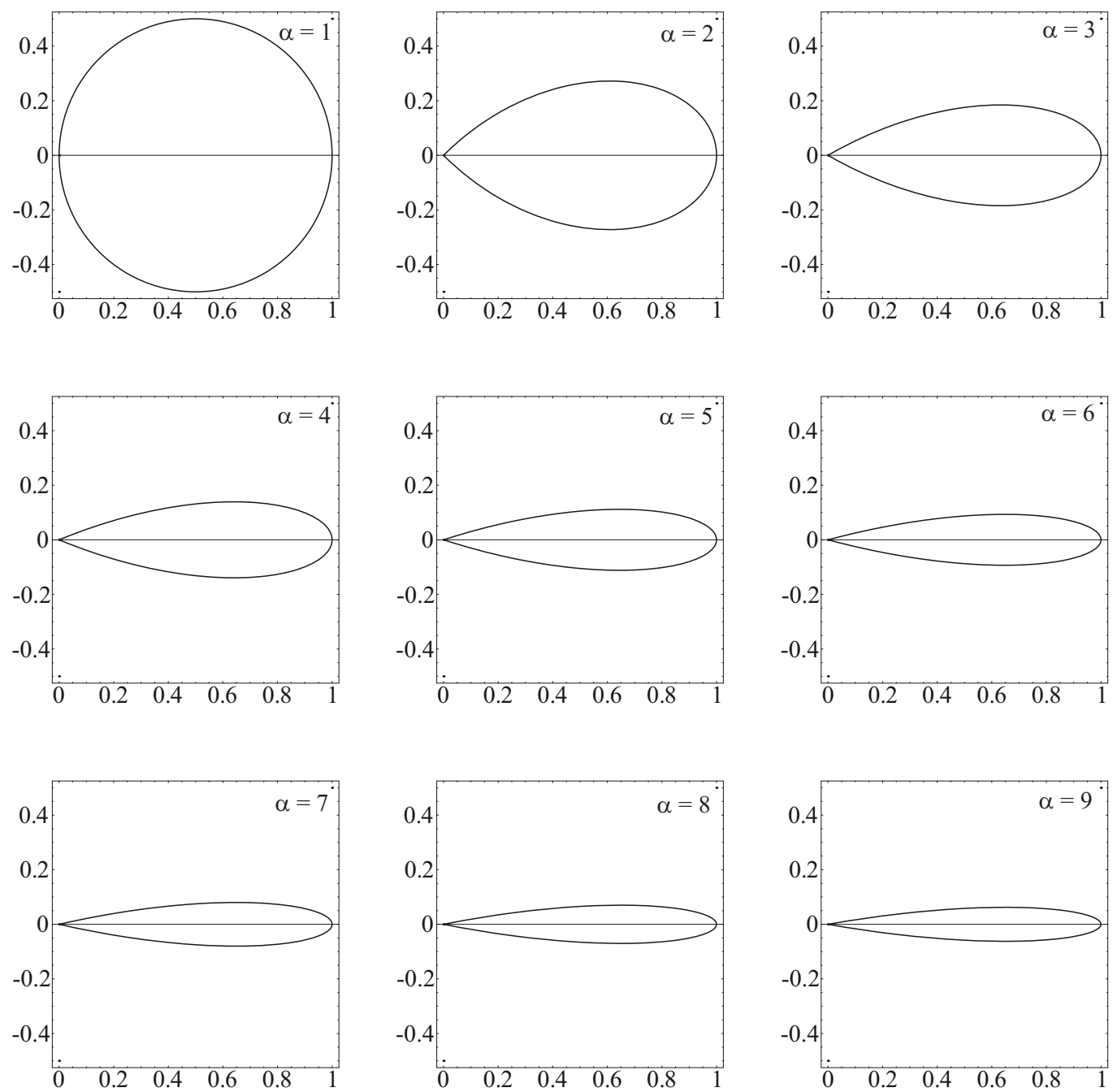

Figure 7: Some examples of the family of functions $r_{i}(c)$ used to take into account the direction of the corridor 

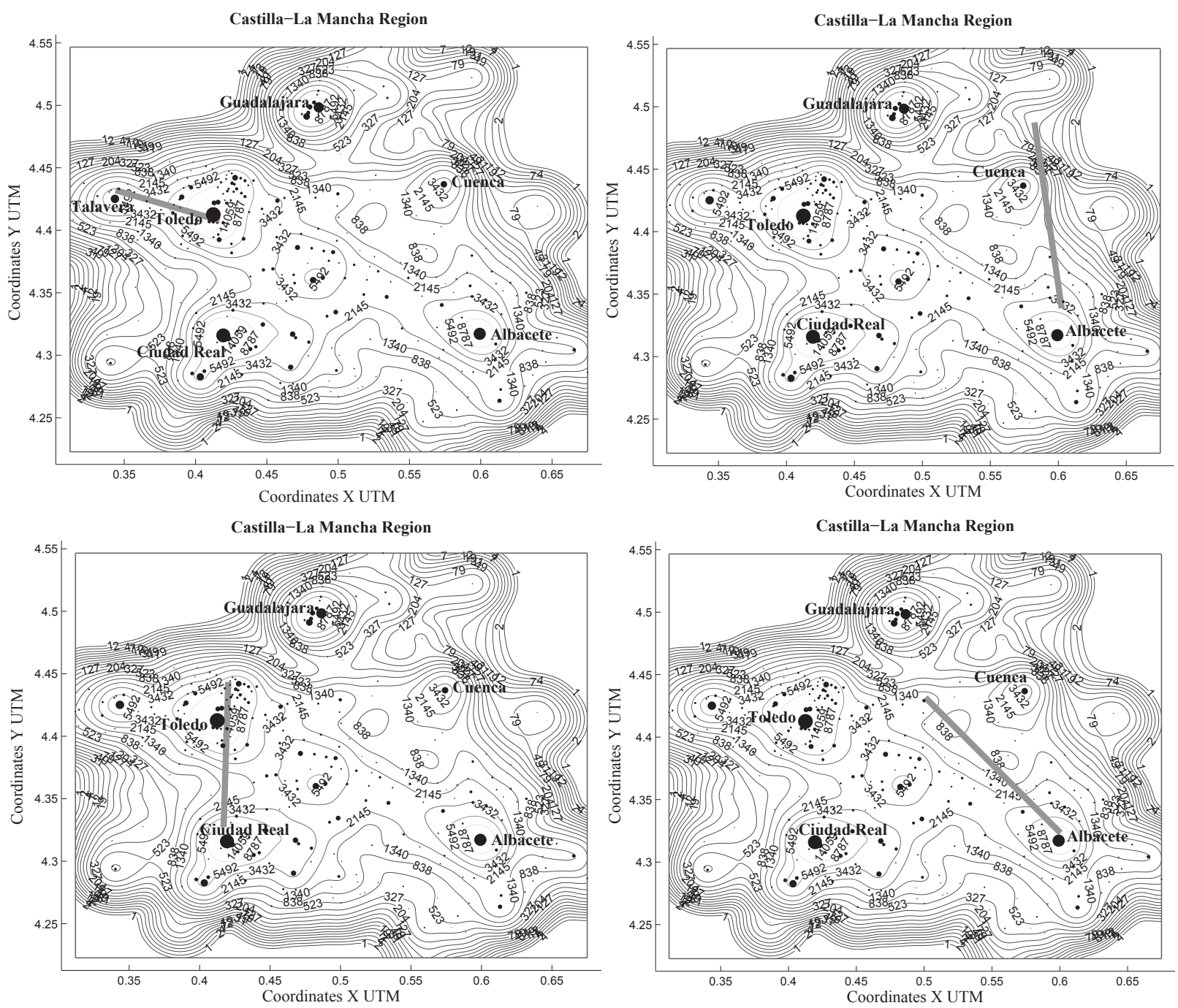

Figure 8: Local optimal solution 
Input. The list of all $(n)$ cities with their coordinates, the list of all $\left(n_{O D}\right)$ OD (origindestination) and the corresponding flows, and a maximum number $\operatorname{Max}_{\text {sol }}$ of initial solutions to be generated.

Output. A set of $n_{\text {sol }}$ initial solutions containing several corridors each if necessary.

Step 0: Initialization. Calculate the traveling levels $\mathcal{I}_{i}$ of all cities and the total flow $O D_{i, j}^{t}=O D_{i, j}+O D_{j, i}$ of each OD (both directions), and sort in increasing values both sets. Let the city index $i=n$, let done $=0$, and $n_{\text {sol }}=1$.

Step 1: City loop. While $i>=1$ and $n_{\text {sol }}<=\operatorname{Max}_{\text {sol }}$, choose the most important city $i i$ in the list, let the OD index $j=n_{O D}$ and continue with Step 2. Otherwise, stop and provide the output information.

Step 2: OD loop. While $j>=1$ and done $=0$, choose the highest flow OD $j j$ in the OD list.

Step 3: Check if OD $j j$ contains city ii. If not, let $j=j-1$ and go to Step 2. Otherwise, continue with Step 4.

Step 4: Check if OD jj contains city ii. Let $n_{\text {sol }}=n_{\text {sol }}+1$, done $=1, i=i-1$, $j=n_{O D}$ and go to Step 1 .

Figure 9: Heuristic initialization 


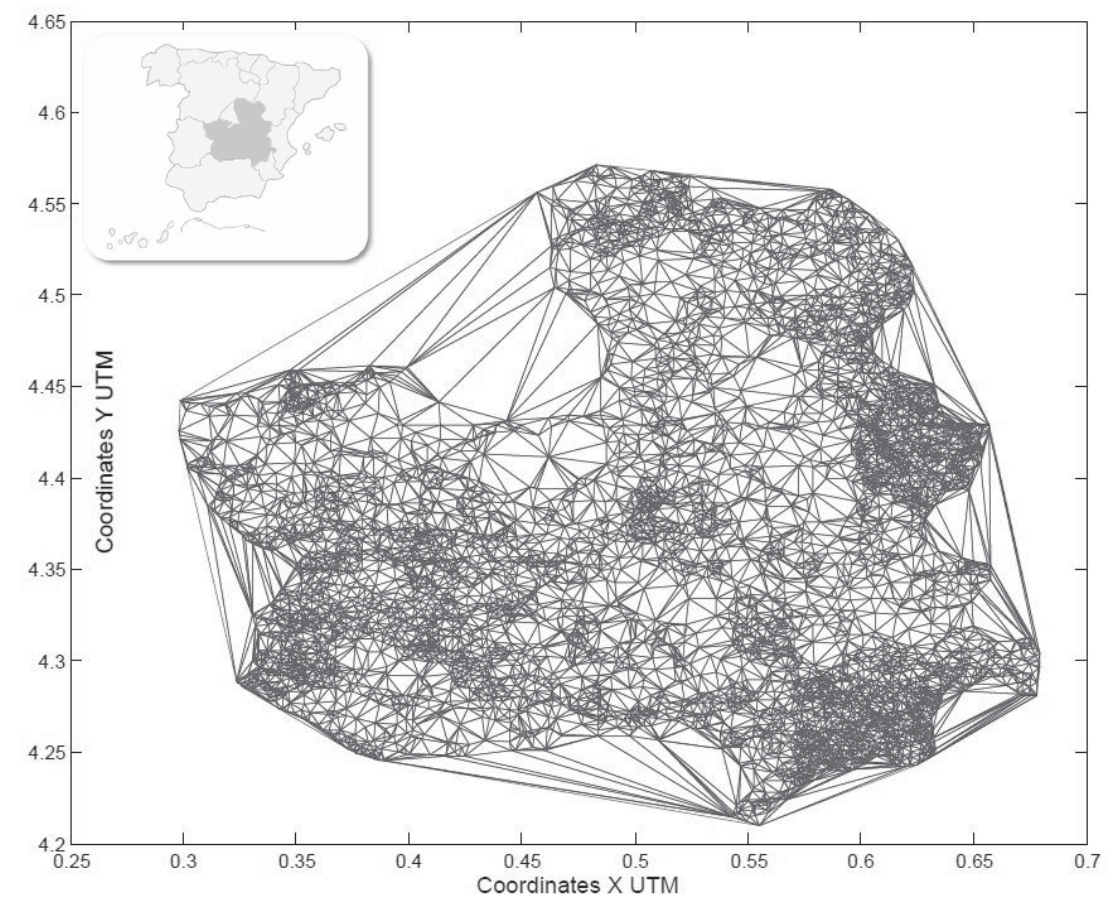

Figure 10: Triangularization of the study region for $B_{1}$
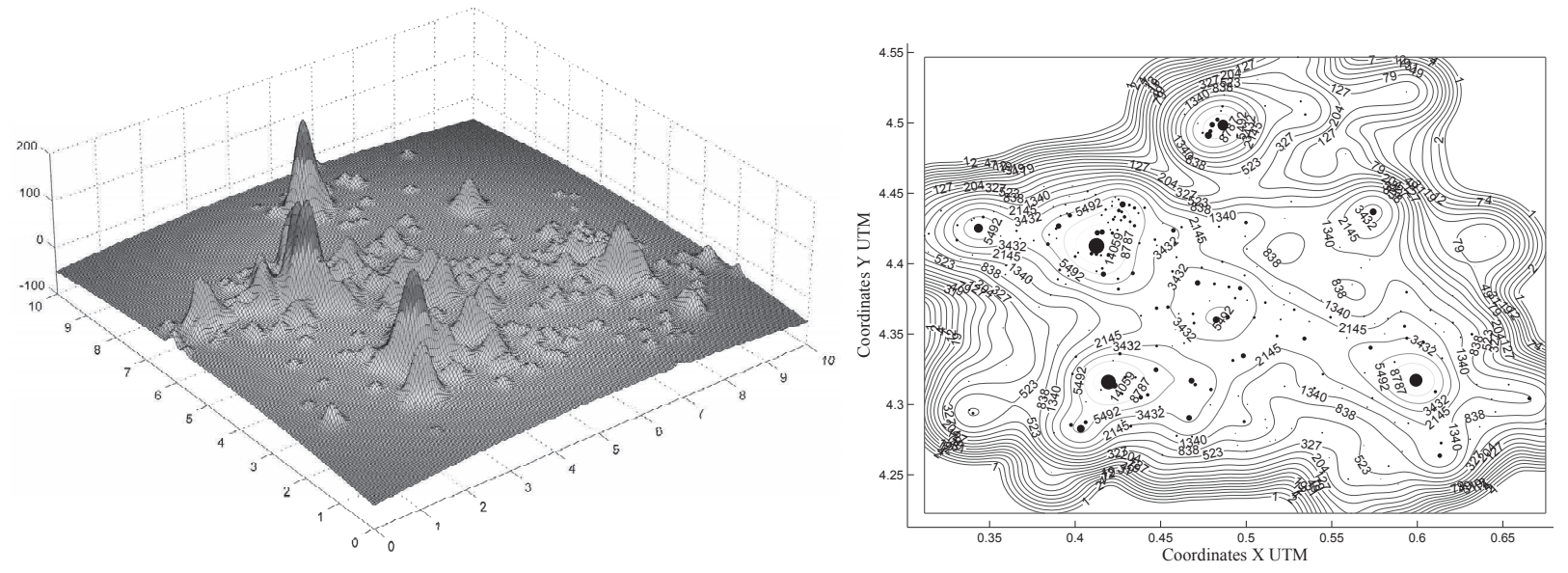

Figure 11: Cost function showing the influence and level curves of the urban and town areas 

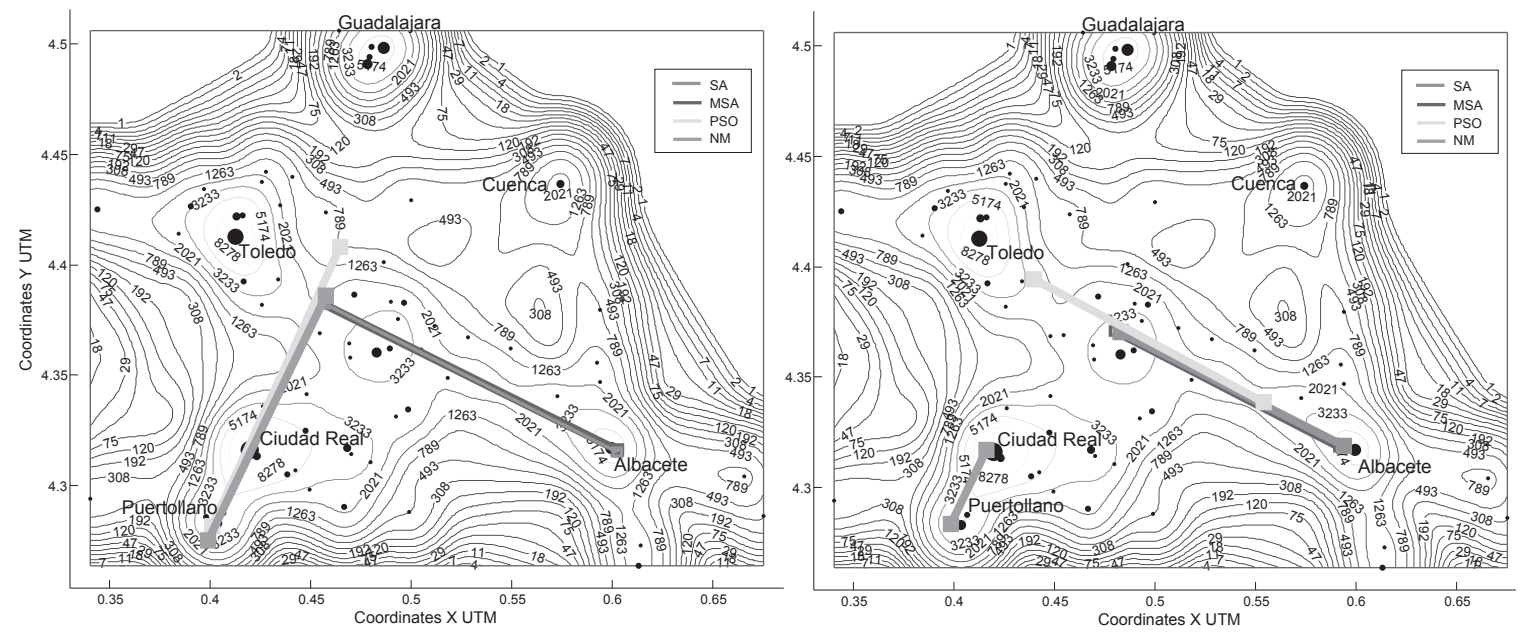

Figure 12: Best solutions obtained with each heuristic algorithm for cost $B_{1}$ and for cost $B_{2}$ 

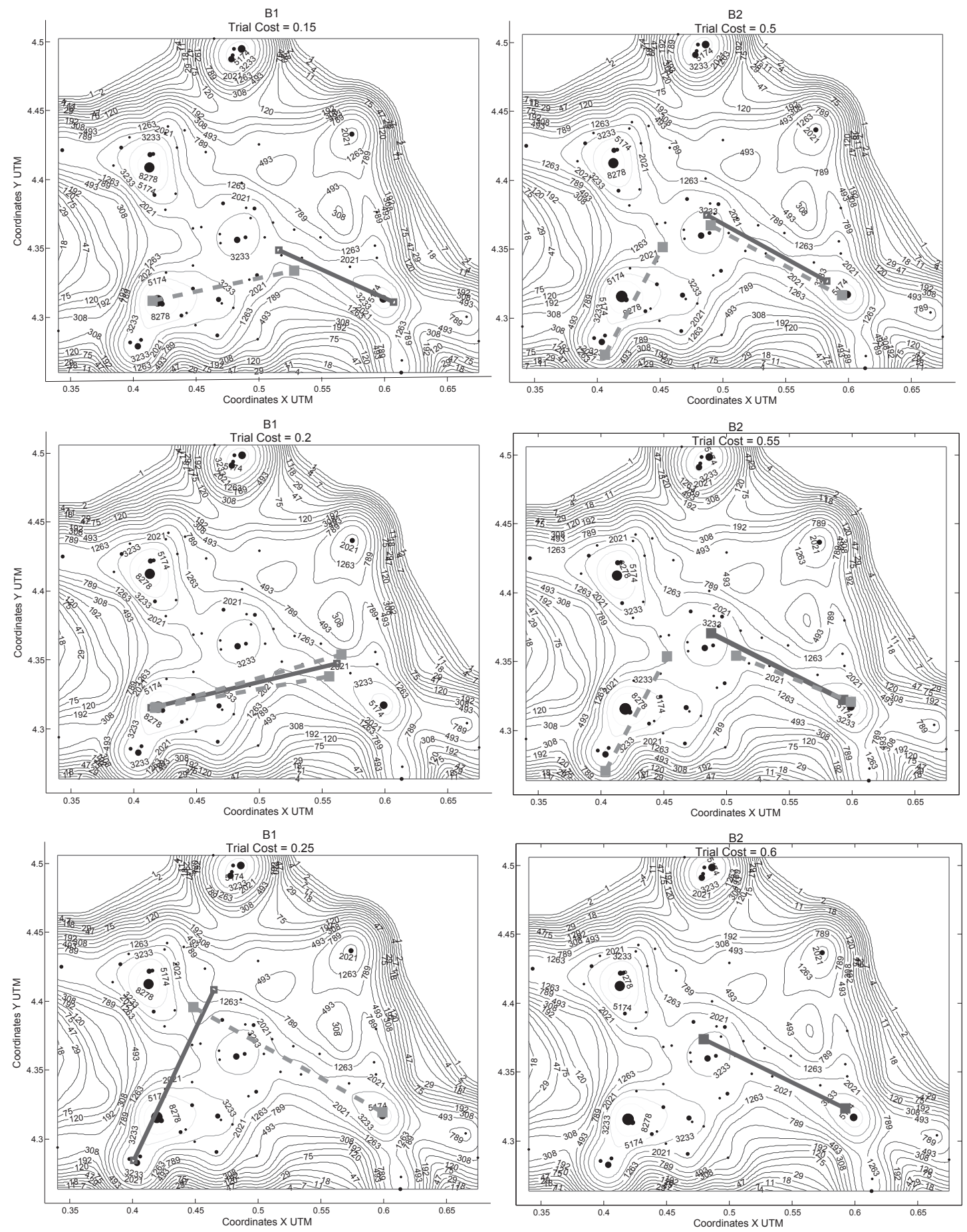

Figure 13: Best solutions with different budgets for cost functions $B_{1}$ and $B_{2}$ 

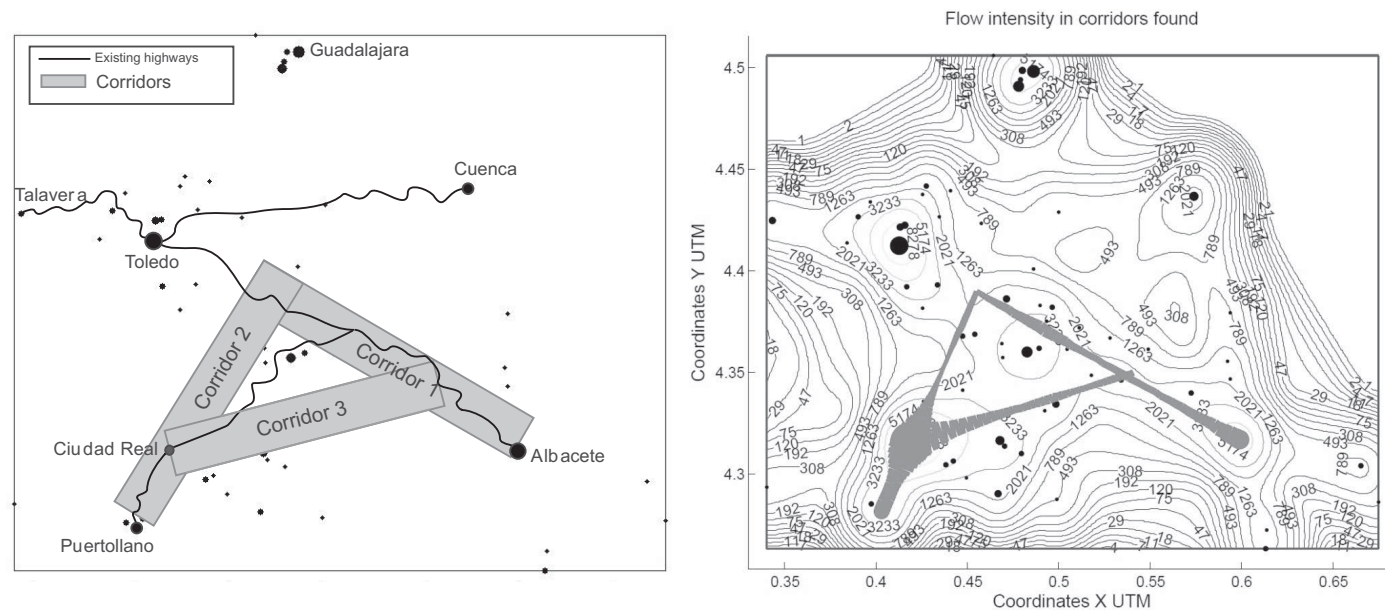

Figure 14: Corridors found
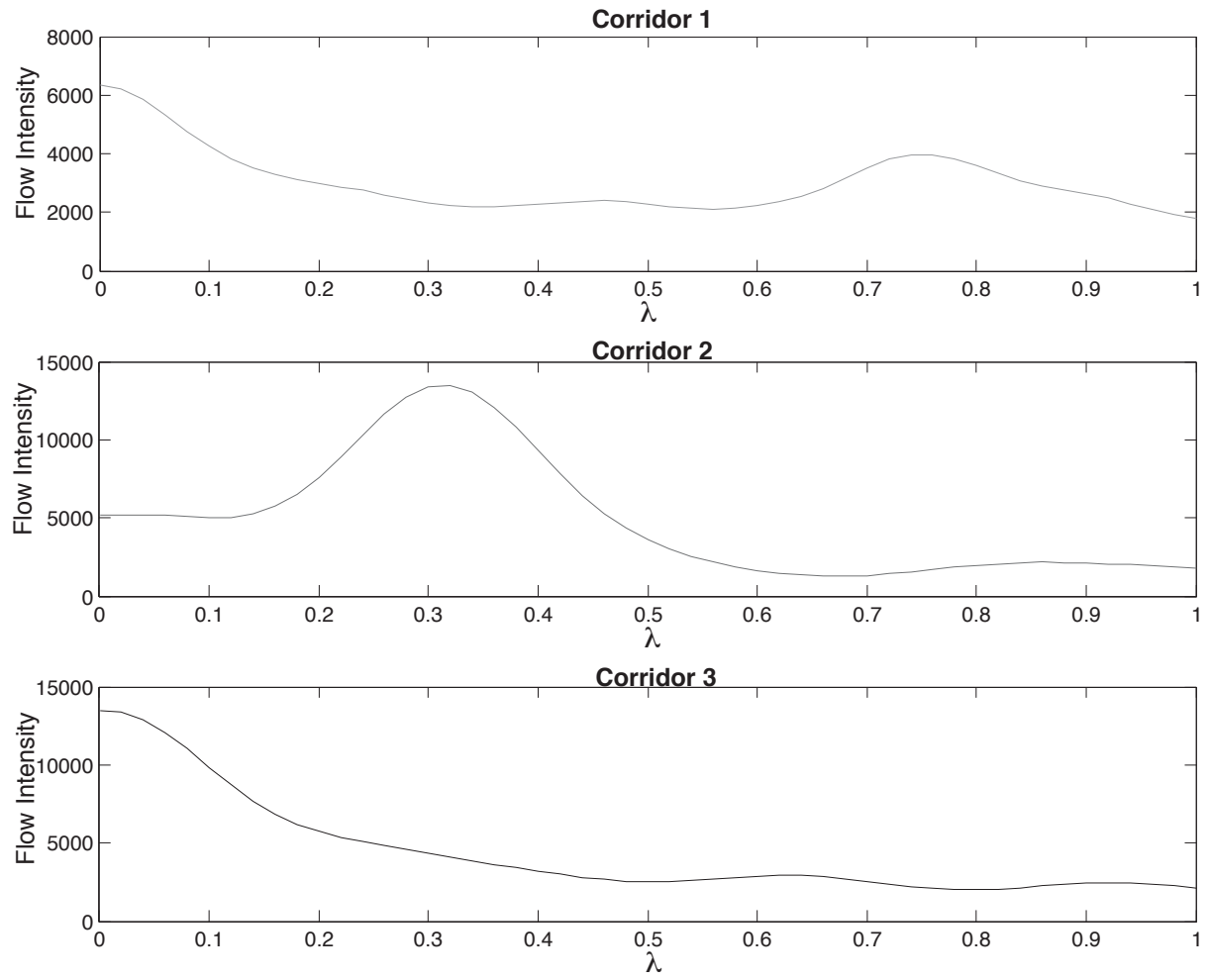

Figure 15: Flow in the corridors found 